\title{
Distinct Doppler Effects for Spontaneously Emitted Photons and Continuously Emitted Waves
}

\author{
Gurcharn S. Sandhu ${ }^{1}$ \\ ${ }^{1}$ Independent Researcher, India \\ Correspondence: Gurcharn S. Sandhu, Independent Researcher, India. E-mail: gurcharn.sandhu@gmail.com
}

Received: June 14, 2017

Accepted: July 18, 2017

Online Published: July 26, 2017

doi:10.5539/apr.v9n4p44

URL: https://doi.org/10.5539/apr.v9n4p44

\begin{abstract}
In this paper, we distinguish between the Doppler effects for spontaneously emitted photons and continuously emitted waves. Under certain plausible assumptions, electron orbits can be modeled for simple atomic systems and such studies show that all permissible electron trajectories correspond to elliptical orbits. From the conservation of energy, momentum and angular momentum, in conjunction with the geometrical model of electron orbits, we derive the Doppler effect for spontaneously emitted photons that is quite different from the one used for continuously generated waves. All astronomical redshifts are currently interpreted by assuming the incoming radiation to be continuously emitted waves. Therefore, widely-observed redshift in radiation from most astronomical sources is interpreted to imply the expanding universe, along with cosmological expansion of space. However, for the spontaneously emitted photons, we show that the photons emitted in forward direction parallel to the emitter velocity get redshifted. That means, the astronomical redshift implies that the emission sources are moving towards the observer and our universe is not expanding. All high redshift astronomical objects are likely to be physically disrupted through dynamic instabilities or explosions and their high redshifts are associated with relativistic shock waves propagating towards the observer. Hence the proposed Doppler effect for the spontaneously emitted photons dismisses the cosmological expansion of space and supports a steady state universe.
\end{abstract}

Keywords: Absolute motion, Doppler effect, electron orbits, photon emission, redshift

\section{Introduction}

The Doppler effect and spectroscopic analysis have played a significant role in the development of our current understanding of the universe. Spectroscopy is the study of absorption and emission frequencies of electromagnetic radiation during its interaction with matter. The Doppler effect refers to change in frequency of a wave associated with relative velocity between the emitter, detector and the wave propagating medium or free space. As per our current understanding, the Doppler effect is associated with continuously emitted waves. However, in this study we are mainly concerned with the phenomenon of spontaneous emission of discrete photons from moving emitters and spontaneous absorption of discrete photons in detectors under various states of their motion. As such our focus in this study will be to examine the Doppler change in photon frequency due to change in absolute motion (Sandhu, 2012) of the emitter or detector. Further we shall also examine whether such change in the frequency of emitted or absorbed photons is also linked to the corresponding changes in the electron transition energies.

\subsection{Electron Transition Energy Levels}

The electron energies of atomic levels are affected by external electric and magnetic fields through their interaction with internal electric and magnetic fields generated by the relative motions of the atomic constituents. In this paper, we investigate whether translational motion of these atoms also affect their electron transition energy levels. For the hydrogen atom, when an electron is moving around the proton in such a way that its electrostatic attraction force towards the proton is in equilibrium with the centrifugal force due to its orbiting motion, the energy state $E_{n}$ of that stable orbital is given by,

$$
E_{n}=-\frac{m_{e} \cdot e^{4}}{8 \varepsilon_{0}^{2} h^{2}} \frac{1}{n^{2}}=-\frac{h c R}{n^{2}}
$$

where $m_{e}$ is mass of electron, $e$ is charge of electron, $c$ is the speed of light, $\varepsilon_{0}$ is permittivity of free space, $\mathrm{h}$ is Planck's constant, and R is the Rydberg constant. The negative value of total energy E indicates that the electron 
is bound to the nucleus whereas at infinite separation of the electron and proton the total energy is defined to be zero. Therefore, the energy $E_{n}$ can be viewed as the binding energy between the electron and the nucleus and represents the amount of energy given out or radiated out of the total energy released due to their electrostatic interaction when the electron is at a finite distance from the nucleus.

When an atomic electron makes a transition between one electron shell of energy $E_{m}$ to another shell of lower energy $E_{n}$, where $m$ and $n$ are shell quantum numbers, the transition is accompanied by the emission of a photon. The frequency of emitted photon is given by $v_{m n}=\left(E_{m}-E_{n}\right) / h$. Alternatively, the atomic electron can absorb a photon of the same frequency $v_{\mathrm{mn}}$ and be promoted from the quantum state of energy $E_{\mathrm{n}}$ to a higher quantum state with energy $E_{m}$. Apart from the shell quantum number $n$, there is a quantum number 1 associated with the electron angular momentum given in the units of reduced Planck constant $\hbar$. Because of its orbital motion around the nucleus, the electron orbit acts as a current loop and thus creates a magnetic field perpendicular to the orbital plane.

Although the gross energies of the electron in hydrogen are fixed by its electrostatic interaction with the nucleus, there are significant magnetic effects on these energies. An electron has an intrinsic magnetic moment and behaves like a tiny bar magnet aligned along its spin axis. The interaction of the electron's magnetic moment with the orbital magnetic field is known as the spin-orbit interaction. The spin-orbit interaction modifies the electron energy proportional to the combination of orbital angular momentum and spin angular momentum. Small differences in energy levels arising from the spin-orbit interaction lead to splitting of spectral lines known as the fine structure. The hyperfine structure is the result of magnetic interactions between the total magnetic moments of the electron and the nucleus and electrostatic interaction between the electric quadrupole moment of the nucleus and the electron.

\subsection{Spectroscopy}

A spectrometer is used to analyze the emitted light in case of emission spectroscopy or transmitted light in the case of absorption spectroscopy. It consists of a disperser that separates the light into its component wavelengths and a system of recording the relative intensities of each of the component wavelengths. The number and arrangement of electrons surrounding their nucleus primarily determine the spectroscopic properties of atoms and ions. Typical energies of electrons within an atom range from a few electron volts $(\mathrm{eV})$ to a few thousand $\mathrm{eV}$. Frequency shifts as small as one part in $10^{15}$ can be observed with high-resolution laser techniques. However, Doppler shifts in frequencies that result from thermal motion of the atoms will broaden any sharp spectral features. Saturation spectroscopy is generally employed for measurement of Doppler-free spectra.

Astronomical redshifts are often detected using spectroscopy or photometry. In the process, continuous intensity of light is monitored as a function of wavelength, to search for specific atomic emission lines whose observed wavelengths can be compared to corresponding measurements on Earth. While spectroscopic redshifts are extremely precise, obtaining good spectra is expensive and time-consuming. Instead, wide-field surveys choose to rely on photometry, which measures the average intensity in a variety of different broad color filters, to obtain data on billions of galaxies. As a result, astronomers rely on photometric redshifts derived from these multi-color imaging data from large populations of galaxies.

\section{Doppler Effect for Continuously Emitted Waves}

As per the current understanding of the Doppler effect, the waves that experience a change in frequency due to motion of emitter or detector are always assumed to be continuously emitted waves. The Doppler effect essentially describes the change in frequency of a wave caused by the motion of the emitter or the observer with respect to the propagation medium or physical space (Roychoudhuri \& Ambroselli, 2013). In case of an emitter it is quite clear that the Doppler induced change in an emitted wave, is the real change in frequency and wavelength of the wave propagating in the propagation medium or space. A moving observer or a detector has no means to determine the frequency or wavelength of the approaching wave until the actual capture or absorption of the wave in the detector. In fact, depending upon the relative velocity of the propagating wave with respect to the moving detector, the number of wavelengths captured in the detector per unit time will vary, giving the impression of a change in frequency of the wave.

\subsection{Doppler Effect due to the motion of Emitter}

Like sound waves, continuously emitted waves are always generated by continuous oscillatory motion of the emitter. While continuous oscillatory motion of a diaphragm generates sound waves in a physical medium, oscillatory motion of electrons in an antenna generates continuous radio waves in free space. Let us assume that an emitter of such waves, when stationary in the wave carrying medium or space, generates continuous waves of frequency $\mathrm{f}_{0}$, time period $\mathrm{T}_{0}$ and wavelength $\lambda_{0}$ that propagate with speed $\mathrm{c}$. Let us consider this emitter moving 
with velocity $\mathbf{u}$ along $\mathrm{X}$-axis while emitting continuous waves in a direction parallel to the velocity vector $\mathbf{u}$. Let $\mathrm{f}_{\text {up }}$ be the frequency and $\lambda_{\text {up }}$ be the wavelength of these waves propagating in space in a direction parallel to the emitter velocity $\mathbf{u}$. During one time period $\mathrm{T}_{0}$ of the emitter oscillator, the leading node of the wave would have travelled a distance $\mathrm{cT}_{0}$ along $\mathrm{X}$-axis, while in the same time period the emitter itself would have travelled a distance of $\mathrm{uT}_{0}$ along $\mathrm{X}$-axis. Hence the trailing node of the wave, emitted at the end of oscillatory time period $\mathrm{T}_{0}$, would get shifted by distance $\mathrm{uT}_{0}$ along $\mathrm{X}$-axis. Therefore,

$$
\lambda_{\text {up }}=\mathrm{cT}_{0}-\mathrm{uT}_{0}=\mathrm{T}_{0}(\mathrm{c}-\mathrm{u})=\frac{\mathrm{c}-\mathrm{u}}{\mathrm{f}_{0}}
$$

Or,

$$
\mathrm{f}_{\mathrm{up}}=\frac{\mathrm{f}_{0}}{(1-\mathrm{u} / \mathrm{c})}
$$

However, if the emitter emits continuous waves in a direction anti-parallel to the velocity vector $\mathbf{u}$, the corresponding frequency $f_{\text {ua }}$ of the waves propagating in a direction opposite to the emitter velocity $\mathbf{u}$ will be obtained by replacing $\mathrm{u}$ with $(-\mathrm{u})$ in equation (2) as,

$$
\mathrm{f}_{\mathrm{ua}}=\frac{\mathrm{f}_{0}}{(1+\mathrm{u} / \mathrm{c})}
$$

\subsection{Doppler Effect due to the Motion of Detector}

Let us now consider the effect of motion of an observer or detector on the frequency and wavelength of absorbed or detected waves. Let $f_{s}$ be the frequency and $\lambda_{s}$ the wavelength of waves propagating along X-axis in physical space at velocity c. A stationary observer or detector will experience or detect unchanged frequency $f_{s}$ on absorption of these waves. However, when the observer or detector moving with velocity $\mathbf{v}$ along $\mathrm{X}$-axis, absorbs these waves propagating in a direction parallel to the detector velocity $\mathbf{v}$, the detected frequency $\mathrm{f}_{\mathrm{dp}} \mathrm{will}$ be different from $\mathrm{f}_{\mathrm{s}}$. Consider one wavelength $\lambda_{\mathrm{s}}$ of the propagating wave just when it is about to make a contact with the detector. When the leading node of the wavelet under consideration makes a contact with the detector, its trailing node is $\lambda_{\mathrm{s}}$ distance behind. By the time the trailing node covers this distance, the detector would have moved forward by a distance proportional to its velocity $\mathbf{v}$ and the total time period $\mathrm{T}_{\mathrm{dp}}$ during which one complete wavelength of the approaching wave is fully captured in the detector. The total distance covered by the trailing node during this time $\mathrm{T}_{\mathrm{dp}}$ is c. $\mathrm{T}_{\mathrm{dp}}$. Therefore,

Or,

$$
\lambda_{\mathrm{s}}+\mathrm{v} \cdot \mathrm{T}_{\mathrm{dp}}=\mathrm{c} . \mathrm{T}_{\mathrm{dp}}
$$

$$
\frac{c}{f_{s}}+\frac{v}{f_{d p}}=\frac{c}{f_{d p}}
$$

Or,

$$
\mathrm{f}_{\mathrm{dp}}=\mathrm{f}_{\mathrm{s}} \frac{\mathrm{c}-\mathrm{v}}{\mathrm{c}}=\mathrm{f}_{\mathrm{s}}(1-\mathrm{v} / \mathrm{c})
$$

However, if the waves to be absorbed are propagating in a direction anti-parallel to the detector velocity vector $\mathbf{v}$, the corresponding detected frequency $f_{\mathrm{da}}$ observed at the detector will be obtained by replacing $\mathrm{v}$ with $(-\mathrm{v})$ in equation (4) as,

$$
\mathrm{f}_{\mathrm{da}}=\mathrm{f}_{\mathrm{s}} \frac{\mathrm{c}+\mathrm{v}}{\mathrm{c}}=\mathrm{f}_{\mathrm{s}}(1+\mathrm{v} / \mathrm{c})
$$

In normal parlance, the waves of frequency $\mathrm{f}_{\mathrm{s}}$ arriving at the detector from behind or parallel direction are said to get down-shifted to frequency $f_{\mathrm{dp}}$ as per equation (4). On the other hand, waves of frequency $\mathrm{f}_{\mathrm{s}}$ arriving at the detector from head-on or anti-parallel direction, are said to get up-shifted to frequency $f_{d a}$ as per equation (5). It may however be emphasized that the down-shifting or up-shifting of space frequency $f_{s}$ does not physically occur until the actual matter-wave interaction during absorption of the wave in the detector.

\subsection{Combined Doppler Effect due to the Motion of Emitter and Detector}

Let us consider an emitter moving along $\mathrm{X}$-axis with velocity $\mathbf{u}$ and a detector also moving ahead along $\mathrm{X}$-axis with velocity $\mathbf{v}$. In the first case, let us assume the emitter emits a beam of continuous waves which propagate along X-axis at velocity $\mathbf{c}$. For $+v e$ values of $\mathbf{u}, \mathbf{v}$ and $\mathbf{c}$, this case corresponds to parallel emission and parallel detection represented by equations (2) and (4) for continuously emitted waves. 


$$
f_{d p}=f_{u p}(1-v / c)=f_{0} \frac{(1-v / c)}{(1-u / c)}
$$

The second case, when both $\mathbf{u}$ and $\mathbf{v}$ are negative, will correspond to anti-parallel emission and anti-parallel detection represented by equations (3) and (5) for continuously emitted waves.

$$
\mathrm{f}_{\mathrm{da}}=\mathrm{f}_{\mathrm{ua}}(1+\mathrm{v} / \mathrm{c})=\mathrm{f}_{0} \frac{(1+\mathrm{v} / \mathrm{c})}{(1+\mathrm{u} / \mathrm{c})}
$$

Similarly, in other specific cases when either $\mathbf{u}$ or $\mathbf{v}$ is negative, the shift in frequency at the detector can be obtained from equation (6) by substituting appropriate values of $u$ and $v$.

The shift in frequency at the detector given by equation (6), can be further simplified by neglecting terms involving $1 / \mathrm{c}^{2}$ and expressing the result in terms of relative receding velocity $\mathbf{w}=(\mathbf{v}-\mathbf{u})$ when $\mathbf{v}>\mathbf{u}$ as,

$$
\mathrm{f}_{\mathrm{d}}=\mathrm{f}_{0}\left(1-\frac{(\mathrm{v}-\mathrm{u})}{\mathrm{c}}\right)=\mathrm{f}_{0}\left(1-\frac{\mathrm{w}}{\mathrm{c}}\right)
$$

However, when the emitter and detector are approaching each other, the relative receding velocity $\mathbf{w}=(\mathbf{v}-\mathbf{u})$ will become negative $(\mathbf{v}<\mathbf{u})$ in equation (8). Since equations (6) and (7) indicate no shift in frequency at the detector when both emitter and detector are moving with a common velocity $\mathbf{u}=\mathbf{v}$, equation (8) with relative receding velocity $\mathbf{w}$ gives the detector frequency for both receding ( $\mathrm{w}$ positive) and approaching ( $\mathrm{w}$ negative) conditions. Here both emitter and detector velocities, $\mathbf{u}$ and $\mathbf{v}$ in equations (6) and (7), are defined with respect to the propagation medium or free space and hence may be viewed as absolute velocities. On the other hand, the receding (or approaching) velocity $\mathbf{w}$ used in equation (8) is only a relative velocity.

In practice, it is extremely difficult to correlate the static emitter frequency $f_{0}$ with the detected frequencies $f_{d p}$ or $\mathrm{f}_{\mathrm{da}}$ through equations (6) and (7), since the absolute emitter and detector velocities $\mathbf{u}$ and $\mathbf{v}$ will invariably be unknown. In contrast equation (8) is practically more convenient since apart from known static emitter frequency $f_{0}$ and the detected frequency $f_{d}$, it involves only one unknown, the relative receding velocity $\mathbf{w}$ which can be easily computed. That is why equation (8) is practically more widely used Doppler equation.

\subsection{Relativistic Doppler Effect}

The relativistic Doppler effect is essentially based on the relative velocity between the emitter and the detector. Taking the relative receding velocity $\mathbf{w}$ as positive, the relativistic Doppler effect is given by a slight modification of equation (8) as,

$$
f_{d}=\gamma_{w}\left(1-\frac{w}{c}\right) f_{0}
$$

Here $\gamma_{\mathrm{w}}$ is the so-called Time dilation factor or the Lorentz factor given by,

$$
\gamma_{\mathrm{w}}=\frac{1}{\sqrt{1-\mathrm{w}^{2} / \mathrm{c}^{2}}}
$$

Combining equations (9) and (10), the relativistic Doppler effect, for +ve receding velocity w, is also given by,

$$
\mathrm{f}_{\mathrm{d}}=\frac{(1-\mathrm{w} / \mathrm{c})}{\sqrt{1-\mathrm{w}^{2} / \mathrm{c}^{2}}} \mathrm{f}_{0}=\frac{\sqrt{1-\mathrm{w} / \mathrm{c}}}{\sqrt{1+\mathrm{w} / \mathrm{c}}} \mathrm{f}_{0}
$$

Since the relative receding velocity is $\mathbf{w}=(\mathbf{v}-\mathbf{u})$, we can isolate the Doppler effect for detector alone moving and emitter stationary at origin $(u=0)$ and replacing $\mathbf{w}$ with $\mathbf{v}$ and $\mathrm{f}_{0}$ with $\mathrm{f}_{\mathrm{s}}$ for parallel absorption in equation $(11)$ as,

$$
\mathrm{f}_{\mathrm{dp}}=\frac{(1-\mathrm{v} / \mathrm{c})}{\sqrt{1-\mathrm{v}^{2} / \mathrm{c}^{2}}} \mathrm{f}_{\mathrm{s}}=\frac{\sqrt{1-\mathrm{v} / \mathrm{c}}}{\sqrt{1+\mathrm{V} / \mathrm{c}}} \mathrm{f}_{\mathrm{s}}
$$

However, if the wave emitted from a stationary emitter is propagating along +ve $\mathrm{X}$-axis and the detector is approaching the wave head-on with velocity $-\mathbf{v}$ then the detected frequency for this anti-parallel absorption is given by,

$$
\mathrm{f}_{\mathrm{da}}=\frac{(1+\mathrm{v} / \mathrm{c})}{\sqrt{1-\mathrm{v}^{2} / \mathrm{c}^{2}}} \mathrm{f}_{\mathrm{s}}=\frac{\sqrt{1+\mathrm{V} / \mathrm{c}}}{\sqrt{1-\mathrm{V} / \mathrm{c}}} \mathrm{f}_{\mathrm{s}}
$$

These two equations (12 and 13) represent the relativistic equivalent of equations (4) and (5) depicting Doppler effect for moving observer or detector. 
A major drawback of this relativistic Doppler effect given by equation (11), is that it fails to account for the separate motions of the emitter or the detector in any Laboratory, or universal reference frame. Since the founding notions of length contraction and time dilation in Relativity have now been attributed to a technical mistake (Sandhu, 2016) in the design and interpretation of Michelson-Morley experiment, the occurrence of $\gamma_{\mathrm{w}}$ factor in Doppler equations needs to be further explored.

\section{Doppler Effect for Spontaneous Photon Emission}

Unlike the continuously emitted waves, photons are not created bit by bit over a period of time but spontaneously released from the wave field accompanying the orbiting electron. The spontaneous emission or absorption of a photon in atoms and molecules is associated with the electron transition between permissible electron orbits. An electron orbit refers to a space-time trajectory of the electron orbiting the parent nucleus or nuclei. However, due to dynamic many-body electromagnetic interactions the electron orbits cannot be precisely fixed in space. Nevertheless, under certain plausible assumptions, detailed electron orbits can still be modeled for simple atomic systems for conceptual understanding of electron trajectories. Such model studies show (Sandhu, 2009) that somewhat akin to Bohr-Sommerfeld atomic model, all permissible electron trajectories correspond to elliptical orbits.

\subsection{Geometry and Dynamic Parameters of Electron Orbits}

During the orbital motion of an electron, its kinetic energy as well as angular velocity increases to a maximum at the perigee and decreases to a minimum at the apogee such that the total angular momentum of the electron remains constant. The sum of its potential $(\mathrm{V})$ and kinetic $(\mathrm{T})$ energies also remain constant equal to its binding energy or the so-called total energy $(\mathrm{E})$. Whereas the principal quantum number $\mathrm{n}$ is linked to the binding energy $\left(\mathrm{E}_{\mathrm{n}}\right)$ of the orbiting electron (equation 1), it also limits the major diameter $\left(\mathrm{d}_{\mathrm{n}}\right)$ of the associated elliptical orbits. The angular momentum quantum number 1 governs the orbital angular momentum $(\mathrm{L}=\mathrm{k} \hbar)$ as well as eccentricity of the electron orbit. In quantum description of atomic orbitals, the angular momentum parameter $\mathrm{k}$ is taken as $\sqrt{ }(1+1)$ but we find it more appropriate to take $\mathrm{k}=(1+1 / 2)$ since it eliminates the controversial situation of zero angular momentum for $\mathrm{s}$ orbitals. The eccentricity $\mathrm{e}_{\mathrm{k}}$ of the electron orbit depends on the angular momentum parameter $\mathrm{k}$ and the principal quantum number $\mathrm{n}$.

$$
\begin{gathered}
e_{k}=\sqrt{1-\left(\frac{k}{n}\right)^{2}} \\
d_{n}=\frac{8 \pi \varepsilon_{0}}{m_{e}}\left(\frac{n \hbar}{e}\right)^{2}
\end{gathered}
$$

Apart from nuclear attractive force acting on the orbiting electron, the total (binding) energy $E_{\mathrm{n}}$ and the angular momentum $\mathrm{k} \hbar$ are the two key parameters that determine the orbit geometry and the associated dynamic characteristics like orbital velocity, angular velocity, kinetic energy, radial and tangential accelerations. Additional interaction energy stored in the orbital and spin magnetic fields of the orbiting electron and the electrostatic interaction energy due to neighboring electrons will effectively increase the total energy $E_{n}$ and hence influence the geometry and dynamic characteristics of the electron orbit.

The varying kinetic energy of the orbiting electron is essentially stored in its accompanying electromagnetic and De Broglie wave fields. During permissible transitions when the orbiting electron experiences maxima of linear acceleration, part of the kinetic energy stored in its accompanying electromagnetic wave field gets dissociated from this compressed wave field and is carried away by the emitted photon. Such dissociation of a part of the kinetic energy of the orbiting electron is governed by the conservation of energy, momentum and angular momentum of the system. Both emission and absorption of a photon results in a change in the total energy $(\Delta \mathrm{E})$ as well as angular momentum $(\Delta \mathrm{L})$ of the orbiting electron leading to a corresponding change in the electron orbit. At any position $r_{i}$ on the orbit, the instantaneous kinetic energy $T_{i}$ and angular velocity $\omega_{i}$ are given by,

$$
\begin{gathered}
\mathrm{T}_{\mathrm{i}}\left(\mathrm{r}_{i}\right)=\frac{\mathrm{e}^{2}}{4 \pi \varepsilon_{0}} \frac{1}{r_{i}}-\left|\mathrm{E}_{\mathrm{n}}\right| \\
\omega_{i}\left(\mathrm{r}_{i}\right)=\frac{k \hbar}{\mathrm{m}_{\mathrm{e}}} \frac{1}{\mathrm{r}_{i}^{2}}
\end{gathered}
$$

The instantaneous velocity $V_{e}$ of the orbiting electron can be split into a radial component $V_{r}$ and a tangential component $V_{t}$ such that its kinetic energy $T_{i}$ can be expressed as $1 / 2 m_{e}\left(V_{r}^{2}+V_{t}^{2}\right)$. The radial component $T_{r}$ of $T_{i}$ is 
much smaller and vanishes at both perigee and apogee points. The tangential component of $T_{i}$ can be expressed in terms of angular velocity $\omega_{\mathrm{i}}$ as,

$$
T_{\omega}=\frac{1}{2} m_{e} r_{i}^{2} \omega_{i}^{2}=\frac{1}{2} L \omega_{i}=\frac{1}{2} k \hbar \omega_{i}
$$

Since the angular momentum $\mathrm{L}$, of the orbiting electron is held constant at a quantized value $\mathrm{k} \hbar$, the rotational kinetic energy $T_{\omega}$ of the electron comes out to be directly proportional to the instantaneous angular speed $\omega_{\mathrm{i}}$. During any transition in electron energy level, since the angular momentum parameter $\mathrm{k}$ changes only by 1 , the corresponding rotational kinetic energy $T_{\omega}$ can change only by $1 / 2 \hbar \omega_{\mathrm{i}}$. As the energy carried away by the emitted photon of angular frequency $\omega$ is $\hbar \omega$, for the instantaneous angular speed $\omega_{\mathrm{i}}=\omega$, half of the photon energy must come from the radial component $\mathrm{T}_{\mathrm{r}}$ of the electron kinetic energy.

\subsection{Phenomenon of Photon Emission from Orbiting Electrons}

The electromagnetic wave field accompanying the orbiting electron consists of time varying electric and magnetic fields. The instantaneous time rate of change of these fields is governed by the angular frequency $\omega$, which in turn is induced by the angular speed $\omega_{\mathrm{i}}$ of the orbiting electron at the retarded instant of their creation in the immediate vicinity of the electron. Starting from the apogee point let the position vector from nucleus to the orbiting electron make an angle $\theta$ with the major axis of the orbit. Orbit simulation studies show that the magnitude of inward radial velocity is maximum at $\theta_{1}=\pi / 2$ and magnitude of linear acceleration of the orbiting electron is maximum at about $\theta_{2}=2 \pi / 3$. Depending upon the magnitude of instantaneous kinetic energy $\left(T_{r}+T_{\omega}\right)$, and linear acceleration of the orbiting electron, emission of a photon in permissible transitions is most likely to occur when the electron is located between $\theta_{1}$ and $\theta_{2}$, where $T_{\mathrm{r}}>1 / 2 \hbar \omega_{\mathrm{i}}$ and $\mathrm{T}_{\mathrm{i}}>\hbar \omega_{\mathrm{i}}$.

The foregoing analysis brings out an important linkage between the change in angular momentum $\Delta \mathrm{L}$ of the orbiting electron and the energy $\mathrm{E}_{\mathrm{ph}}$ of the emitted photon. Angular momentum $\mathrm{L}$ of the electron orbiting the nucleus is inherently associated with the relative motion of the electron with respect to the nucleus. Any common uniform translational motion of the nucleus-electron system or the emitter atom or molecule will not affect the angular momentum $\mathrm{L}$ of the orbiting electron. To eject or emit a portion of the kinetic energy of the orbiting electron, it is necessary to affect a corresponding change in the angular momentum of the electron. However, to induce a change in the angular momentum $\mathrm{L}$ of the orbiting electron, it is necessary to affect a change in the orbital momentum $p_{r}$ relative to the nucleus.

When the orbiting electron approaches its point of maximum acceleration, the accompanying wave field gets compressed into a sort of transient shock wave. The photon being emitted is spontaneously released from this transient shock wave field. Separation of the radiation wave field from the attached wave field is governed by a transient reaction impulse that induces a change in orbital momentum $\Delta \mathrm{p}_{\mathrm{r}}$ with respect to the nucleus. It is this change in relative momentum $\Delta \mathrm{p}_{\mathrm{r}}$ of the electron that governs the energy content of the emitted wave packet through a corresponding change in angular momentum $\Delta \mathrm{L}$ of the orbiting electron. As per our current understanding, change in orbital momentum $\Delta \mathrm{p}_{\mathrm{r}}$ is viewed as a recoil momentum, a consequence of the photon emission, whereas it needs to be viewed as an internal ejection impulse for emission of the photon from the kinetic energy of an orbiting electron.

When the emitting atom is at absolute rest, whole energy $\mathrm{E}_{0}$ of the spontaneously emitted photon is extracted from the intrinsic kinetic energy of the orbiting electron and is counted as the electron transition energy. During the emission of a photon, recoil momentum $\Delta \mathrm{p}_{\mathrm{r}}$ is experienced by the orbiting electron and its parent nucleus which is interlinked with the change in their angular momentum. Any change in angular momentum of the orbiting electron will induce a corresponding change in the shape, size and orientation of the electron orbit as well as its total energy. As such, any change in recoil momentum intricately affects the corresponding electron transition energy. If $v_{0}$ is the frequency and $\mathrm{p}_{0}$ is the momentum of the photon emitted from a light source at rest, then photon energy $E_{0}$ is given by,

$$
\mathrm{E}_{0}=\hbar \omega_{0}=\mathrm{h} v_{0}=\mathrm{p}_{0} \mathrm{c} .
$$

And

$$
\Delta \mathrm{p}_{\mathrm{r}}=-\mathrm{p}_{0}
$$

\subsection{Doppler Effect due to Photon Emission from Moving Emitter}

A light emitting atom moving along $\mathrm{X}$-axis will experience a recoil momentum while emitting a photon of momentum $\mathbf{p}_{0}$ along $\mathrm{X}$-axis. The recoil first originates as a change in orbital momentum $\Delta \mathrm{p}_{\mathrm{r}}$ and then gets transferred to the nucleus through the mechanism of change in angular momentum $\Delta \mathrm{L}$. Consider the case when 
emitter nucleus and the orbiting electron both experience a common translational motion with absolute velocity $\mathbf{u}$ along X-axis. The component of kinetic energy associated with uniform translational velocity $\mathbf{u}$ of the orbiting electron cannot be emitted out or dissipated until the electron encounters a strong acceleration or deceleration in its translational motion. The strong acceleration experienced by the orbiting electron in its rotational motion will only enable it to release a portion of its rotational kinetic energy proportional to the change in its angular momentum $\Delta \mathrm{L}$. Any elemental energy content $\delta \mathrm{E}$ belonging to the nucleus-electron system will possess a linear momentum $\left(\delta \mathrm{E} / \mathrm{c}^{2}\right) \cdot \mathbf{u}$ associated with absolute uniform velocity $\mathbf{u}$. Therefore, when a small energy content $\delta \mathrm{E}$ is being emitted as a photon from the wave field accompanying the orbiting electron then to impart it a momentum $\delta \mathrm{E} / \mathrm{c}$, the additional momentum required to be imparted to this energy content $\delta \mathrm{E}$ is given by,

$$
\left[\frac{\delta \mathrm{E}}{c^{2}}(c-u)\right]=\frac{\delta \mathrm{E}}{c}(1-\mathrm{u} / \mathrm{c})=-\Delta p_{r}
$$

That is, the magnitude of ejection impulse or the change in orbital momentum $\Delta \mathrm{p}_{\mathrm{r}}$ gets reduced by a factor (1-u/c) when the photon is emitted from an atom moving with velocity $\mathrm{u}$ in the direction of photon emission. The change in orbital momentum $\Delta \mathrm{p}_{\mathrm{r}}$ is directly associated with the corresponding change in its angular momentum $\Delta \mathrm{L}$, leading to the electron transition to a new orbit. The magnitude of this change in its angular momentum $\Delta \mathrm{L}$ governs the emitted photon energy and the transition energy of the orbiting electron. As such, when the magnitude of ejection impulse and hence change in angular momentum $\Delta \mathrm{L}$ gets reduced by a factor of $(1-\mathrm{u} / \mathrm{c})$ due to the uniform motion of emitting atom, the magnitude of electron transition energy $\Delta \mathrm{E}_{\mathrm{n}}{ }^{\prime}$ must also get reduced from the static transition energy $\Delta \mathrm{E}_{\mathrm{n}}$ by the same factor $(1-\mathrm{u} / \mathrm{c})$.

$$
\Delta E_{n}^{\prime}=\Delta \mathrm{E}_{\mathrm{n}}\left(1-\frac{\mathrm{u}}{\mathrm{c}}\right)
$$

Hence, energy $E_{u}$ of the photon emitted from a moving light source will be less than the energy $E_{0}$ of the corresponding photon emitted from a stationary light source, by a factor of $(1-\mathrm{u} / \mathrm{c})$.

$$
\mathrm{E}_{\mathrm{u}}=\mathrm{E}_{0} \cdot\left(1-\frac{\mathrm{u}}{\mathrm{c}}\right)=\mathrm{p}_{0} \cdot(\mathrm{c}-\mathrm{u})
$$

Further, the momentum $\mathrm{p}_{\mathrm{u}}$ of the photon emitted from a moving light source can be related to the momentum $\mathrm{p}_{0}$ of the corresponding photon emitted from a stationary light source as,

$$
\mathrm{p}_{\mathrm{u}}=\mathrm{p}_{0} \cdot\left(1-\frac{\mathrm{u}}{\mathrm{c}}\right)
$$

However, on the consideration of energy conservation during photon emission from a moving emitter, equations (23) and (24) will get modified by a relativistic or dynamic mass factor $\gamma_{\mathrm{u}}$ given by,

$$
\gamma_{u}=\frac{1}{\sqrt{1-u^{2} / c^{2}}}
$$

The dynamic mass-energy content of the moving emitter atom as well as of the orbiting electron gets increased by dynamic mass factor $\gamma_{\mathrm{u}}$. As per equation (1), the electron transition energy $\Delta \mathrm{E}_{\mathrm{n}}{ }^{\prime}$ must also depend on the dynamic mass of the orbiting electron. Hence, the energy content emitted in the form of photon will also get increased by the dynamic factor $\gamma_{u}$. Therefore, equations (23) and (24) for the energy content $E_{u}$ and the momentum $p_{u}$ of the photon emitted in space in forward direction, will get modified by the dynamic mass factor, or the so-called Lorentz factor $\gamma_{\mathrm{u}}$ as,

$$
\mathrm{p}_{\mathrm{u}}=\mathrm{p}_{0} \cdot \gamma_{\mathrm{u}}\left(1-\frac{\mathrm{u}}{\mathrm{c}}\right)
$$

And

$$
\begin{gathered}
E_{u}=p_{u} \cdot c=E_{0} \cdot \gamma_{u}\left(1-\frac{u}{c}\right) \\
v_{u}=v_{0} \cdot \gamma_{u}\left(1-\frac{u}{c}\right)=\frac{\left(1-\frac{u}{c}\right)}{\sqrt{1-u^{2} / c^{2}}} v_{0}
\end{gathered}
$$

Here, $v_{u}$ is the frequency of the photon emitted from a moving light source in forward direction and $v_{0}$ is the frequency of the corresponding photon emitted from a stationary light source. Similarly, equation (22) will get modified to, 


$$
\Delta E_{n}^{\prime}=\Delta \mathrm{E}_{\mathrm{n}} \cdot \gamma_{\mathrm{u}}\left(1-\frac{\mathrm{u}}{\mathrm{c}}\right)=\frac{\left(1-\frac{\mathrm{u}}{\mathrm{c}}\right)}{\sqrt{1-\mathrm{u}^{2} / \mathrm{c}^{2}}} \Delta \mathrm{E}_{\mathrm{n}}
$$

This situation can be generalized for different angles of inclination between light source velocity vector and the direction of photon emission. When a light source, moving at velocity $\mathbf{u}$ along $\mathrm{X}$-axis, emits a photon at a relative emission velocity $\mathbf{V}_{\mathbf{e}}$ inclined at an angle $\theta$ to the velocity vector $\mathbf{u}$ (Figure 1), the resultant photon velocity $\mathbf{c}$ is a vector sum of $\mathbf{u}$ and $\mathbf{V}_{\mathbf{e}}$ such that,

Or,

$$
\begin{gathered}
\boldsymbol{V}_{\boldsymbol{e}}=\boldsymbol{c}-\boldsymbol{u} \\
V_{e}=\sqrt{c^{2}-u^{2} \sin ^{2} \theta}-u \cos \theta
\end{gathered}
$$

For a given electron transition, the transition energy $\Delta \mathrm{E}_{\mathrm{n}}$ and the energy of a photon emitted from a moving light source varies with relative emission velocity $\mathrm{V}_{\mathrm{e}} / \mathrm{c}$ apart from the relativistic or dynamic factor $\gamma_{\mathrm{u}}$.

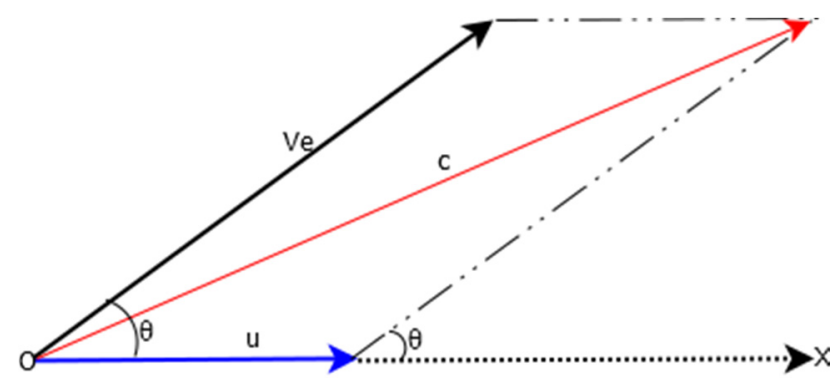

Figure 1. Relative emission velocity $\mathbf{V}_{\mathbf{e}}$ of photon. $\mathrm{c}^{2}=\mathrm{u}^{2}+\mathrm{V}_{\mathrm{e}}^{2}+2 \mathrm{u} \mathrm{V}_{\mathrm{e}} \operatorname{Cos}(\theta)$

When a photon is emitted forward or parallel to the light source velocity $\mathbf{u}$, the relative emission velocity $V_{\text {ep }}$ is obtained from equation (31) with angle $\theta=0$, as

$$
V_{e p}=c-u
$$

When a photon is emitted backwards or anti-parallel to the light source velocity $\mathbf{u}$, the relative emission velocity $\mathrm{V}_{\text {ea }}$ is obtained from equation (31) with angle $\theta=\pi$, as

$$
V_{e a}=c+u
$$

However, when a photon is emitted in a direction transverse to the light source velocity $\mathbf{u}$, the relative emission velocity $\mathrm{V}_{\mathrm{et}}$ is obtained from equation (31) with angle $\theta=\pi / 2$, as

$$
\mathrm{V}_{\mathrm{et}}=\sqrt{\mathrm{c}^{2}-\mathrm{u}^{2}}
$$

We can rewrite equation (26) to express the momentum $p_{u}$ of photon propagating in free space, after emission from a moving light source, in terms of relative emission velocity $\mathrm{V}_{\mathrm{e}} / \mathrm{c}$ as,

$$
\mathrm{p}_{\mathrm{u}}=\mathrm{p}_{0} \gamma_{\mathrm{u}} \frac{\mathrm{v}_{\mathrm{e}}}{\mathrm{c}}
$$

The photon energy $E_{u}$, wavelength $\lambda_{u}$ and frequency $v_{u}$ can be correlated with its momentum $p_{u}$ in free space through standard relations using Planck's constant $h$ as,

$$
\begin{gathered}
E_{u}=p_{u} c \quad \lambda_{u}=\frac{h}{p_{u}} \\
v_{u}=\frac{p_{u} c}{h}=\gamma_{u} \frac{v_{e}}{c} v_{0}
\end{gathered}
$$

When a photon is emitted forward or parallel to the light source velocity $\mathbf{u}$, replacing the relative emission velocity $\mathrm{V}_{\mathrm{e}}$ by $\mathrm{V}_{\mathrm{ep}}$, the frequency $v_{\mathrm{up}}$ of the emitted photon propagating in free space is given by,

$$
v_{\text {up }}=\gamma_{\mathrm{u}} \frac{\mathrm{vep}_{\mathrm{ep}}}{\mathrm{c}} v_{0}=\frac{\left(1-\frac{\mathrm{u}}{\mathrm{c}}\right)}{\sqrt{1-\mathrm{u}^{2} / \mathrm{c}^{2}}} v_{0}
$$

Here, $v_{0}$ is the standard reference frequency of the photon emitted under static condition for the given transition. When a photon is emitted backwards or anti-parallel to the light source velocity $\mathbf{u}$, replacing the relative emission velocity $\mathrm{V}_{\mathrm{e}}$ by $\mathrm{V}_{\mathrm{ea}}$, the frequency $v_{\text {ua }}$ of photon propagating in free space is given by, 


$$
v_{\mathrm{ua}}=\gamma_{\mathrm{u}} \frac{\mathrm{v}_{\mathrm{ea}}}{\mathrm{c}} v_{0}=\frac{\left(1+\frac{\mathrm{u}}{\mathrm{c}}\right)}{\sqrt{1-\mathrm{u}^{2} / \mathrm{c}^{2}}} v_{0}
$$

Similarly, when a photon is emitted in a direction transverse to the light source velocity $\mathbf{u}$, replacing the relative emission velocity $\mathrm{V}_{\mathrm{e}}$ by $\mathrm{V}_{\mathrm{et}}$, the frequency $\mathrm{v}_{\mathrm{ut}}$ of photon propagating in free space is given by,

$$
v_{\mathrm{ut}}=\gamma_{\mathrm{u}} \frac{\mathrm{v}_{\mathrm{et}}}{\mathrm{c}} v_{0}=v_{0}
$$

The occurrence of $\gamma_{\mathrm{u}}$ factor in the above equations can be attributed to the dynamic increase in rest mass $\mathrm{m}_{\mathrm{e}}$ of electron with velocity $\mathbf{u}$ of the emitter. The frequency of a photon emitted during an orbital transition of the electron is directly proportional to mass $m_{e}$ of the electron. Therefore, the frequency of a photon emitted from an atom or molecule moving with velocity $\mathbf{u}$ will also increase by the factor $\gamma_{\mathrm{u}}$. Above equations imply that the emission frequency of an emitter in motion becomes direction sensitive or anisotropic with the emitter velocity $\mathbf{u}$. Thus, the Doppler change in frequency with the motion of emitter may be attributed to an inherent change in electron transition energy leading to the change in frequency of the emitted photons. The change in electron transition energy may be further attributed to the change in photon ejection impulse and the corresponding electron orbit parameters, with motion of the emitter.

\section{Doppler Effect for Spontaneous Photon Absorption}

\subsection{Doppler Effect due to Photon Absorption in a Moving Detector}

Let us now examine the change in photon absorption process in an atom or molecule in motion. As in the case of emission, there may be a corresponding anisotropic change in photon absorption in a moving detector. Since the inherent electron transition is reversible for absorption and emission of photons, as implicit in the optical resonance experiments, the transition energy between two particular states ' 1 ' and ' 2 ' associated with absorption $E_{12}$ and emission $\mathrm{E}_{21}$ must be equal. Therefore, transition energy between two states 1 and 2 must also be equally anisotropic for absorption $E_{12}^{\prime}$ and emission $E_{21}^{\prime}$ in moving detectors and emitters. Since the anisotropic change during emission is proportional to the relative emission velocity $\mathbf{V}_{\mathbf{e}}$, the corresponding anisotropic change during absorption may also be proportional to the relative capture velocity $\mathbf{V}_{\mathbf{c}}=\mathbf{c}-\mathbf{u}$ (figure 2).

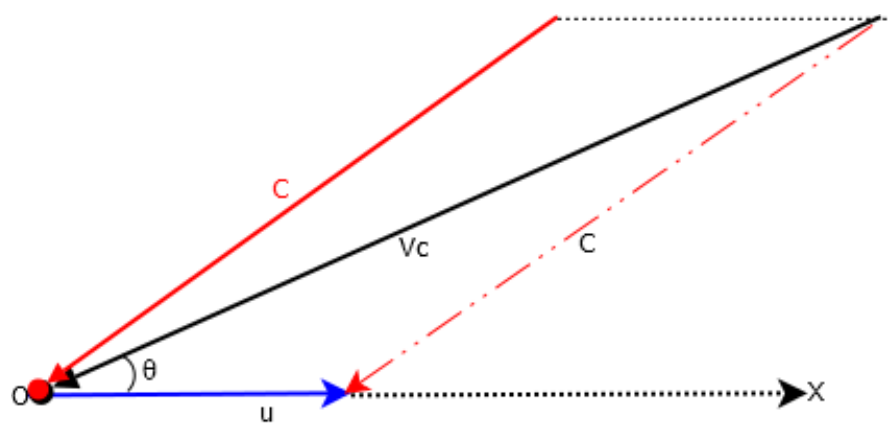

Figure 2. Relative capture velocity $\mathbf{V}_{\mathbf{c}}$ of photon. $\quad \mathrm{V}_{\mathrm{c}}=\mathrm{u} \cdot \operatorname{Cos}(\theta)+\sqrt{ }\left(\mathrm{c}^{2}-\mathrm{u}^{2} \operatorname{Sin}^{2}(\theta)\right)$

When a detector, moving at velocity $\mathbf{u}$ along $\mathrm{X}$-axis, absorbs a photon at a relative capture velocity $\mathbf{V}_{\mathbf{c}}$ inclined at an angle $\theta$ to the velocity vector $\mathbf{u}$, the relative capture velocity $\mathbf{V}_{\mathbf{c}}$ is given by,

Or,

$$
\begin{gathered}
\mathbf{V}_{\mathbf{c}}=\mathbf{c}-\mathbf{u} \\
\mathrm{V}_{\mathrm{c}}=\sqrt{\mathrm{c}^{2}-\mathrm{u}^{2} \sin ^{2} \theta}+\mathrm{u} \cos \theta
\end{gathered}
$$

When a photon is captured head-on from a light beam propagating anti-parallel to the detector velocity $\mathbf{u}$, the relative capture velocity $\mathrm{V}_{\mathrm{ca}}$ is obtained from equation (39) with angle $\theta=0$, as

$$
\mathrm{V}_{\mathrm{ca}}=\mathrm{c}+\mathrm{u}
$$

When a photon is captured from a light beam propagating parallel to the detector velocity $\mathbf{u}$, the relative capture velocity $V_{c p}$ is obtained from equation (39) with angle $\theta=\pi$, as

$$
\mathrm{V}_{\mathrm{cp}}=\mathrm{c}-\mathrm{u}
$$

Consider an electron transition under which a photon of frequency $v_{0}$ is emitted in static condition and a photon of frequency $v_{\text {up }}$ emitted in forward direction from a moving emitter as per equation (35). Let this photon be turned 
around to become a part of a laser beam of frequency $v_{\mathrm{sa}}\left(=v_{\mathrm{up}}\right)$ propagating in free space. If it is absorbed head-on by the moving emitter/detector, then it must induce a reverse electron transition of the same type as originally caused by the absorption of a photon of frequency $v_{0}$ under static conditions. That is,

Therefore,

$$
v_{\mathrm{sa}}=v_{\mathrm{up}}=\gamma_{u}\left(1-\frac{\mathrm{u}}{\mathrm{c}}\right) v_{0}=\frac{\left(1-\frac{\mathrm{u}}{\mathrm{c}}\right)}{\sqrt{1-\mathrm{u}^{2} / \mathrm{c}^{2}}} v_{0}
$$

Or,

$$
v_{0}=\frac{\sqrt{1-\mathrm{u}^{2} / \mathrm{c}^{2}}}{\left(1-\frac{\mathrm{u}}{\mathrm{c}}\right)} v_{\mathrm{sa}}=\frac{\left(1+\frac{\mathrm{u}}{\mathrm{c}}\right)}{\sqrt{1-\mathrm{u}^{2} / \mathrm{c}^{2}}} v_{\mathrm{sa}}=\gamma_{u}\left(1+\frac{\mathrm{u}}{\mathrm{c}}\right) v_{\mathrm{sa}}
$$

$$
v_{0}=\gamma_{u}\left(1+\frac{\mathrm{u}}{\mathrm{c}}\right) v_{\mathrm{sa}}=\gamma_{u} \frac{\mathrm{v}_{\mathrm{ca}}}{\mathrm{c}} \nu_{\mathrm{sa}}
$$

Here we distinguish the photon emission and absorption frequencies by using $v_{u}$ for the frequency of emitted photons, $v_{\mathrm{s}}$ for the frequency of photons propagating in free space before their capture by the detectors and $v_{\mathrm{d}}$ for the effective detected frequency. Equation (40B) represents the head-on or anti-parallel absorption of a photon of frequency $v_{\mathrm{sa}}$ to induce an electron transition of the same type as normally induced by a photon of frequency $v_{0}$ under static condition. Therefore, the effective detected frequency $v_{\mathrm{d}}$ for a photon of space frequency $v_{\mathrm{sa}}$ absorbed under head-on or anti-parallel motion of the detector will be given by,

$$
v_{\mathrm{d}}=v_{0}=\gamma_{u} \frac{\mathrm{v}_{\mathrm{ca}}}{\mathrm{c}} v_{\mathrm{sa}}
$$

Now consider a photon of frequency $v_{\text {ua }}$ given by equation (36) emitted in backward direction. If this photon is turned around and forms a part of a laser beam of frequency $v_{\mathrm{sp}}=v_{\mathrm{ua}}$ and gets absorbed while propagating parallel to the moving emitter/detector, then it must induce a reverse electron transition of the same type as originally caused by the absorption of a photon of frequency $v_{0}$ under static condition. That is,

Therefore,

$$
\begin{gathered}
v_{\mathrm{sp}}=v_{\mathrm{ua}}=\gamma_{u}\left(1+\frac{\mathrm{u}}{\mathrm{c}}\right) v_{0}=\frac{\left(1+\frac{\mathrm{u}}{\mathrm{c}}\right)}{\sqrt{1-\mathrm{u}^{2} / \mathrm{c}^{2}}} v_{0} \\
v_{0}=\frac{v_{\mathrm{sp}} \sqrt{1-\mathrm{u}^{2} / \mathrm{c}^{2}}}{\left(1+\frac{\mathrm{u}}{\mathrm{c}}\right)}=\frac{v_{\mathrm{sp}} \sqrt{1-\mathrm{u} / \mathrm{c}}}{\sqrt{1+\mathrm{u} / \mathrm{c}}}=\gamma_{u}\left(1-\frac{\mathrm{u}}{\mathrm{c}}\right) v_{\mathrm{sp}} \\
v_{\mathrm{d}}=v_{0}=\gamma_{u}\left(1-\frac{\mathrm{u}}{\mathrm{c}}\right) v_{\mathrm{sp}}=\gamma_{u} \frac{\mathrm{v}_{\mathrm{cp}}}{\mathrm{c}} v_{\mathrm{sp}}
\end{gathered}
$$

Equations (40C) and (41B) represent the absorption of photons of frequency $v_{\mathrm{sa}}$ from anti-parallel direction and frequency $v_{\mathrm{sp}}$ from parallel direction in a moving detector. We can rewrite these equations as detector equations by replacing static absorption frequency $v_{0}$ with effective detected frequency $v_{\mathrm{d} 0}$ and detector velocity $\mathrm{u}$ with $\mathrm{v}$ as,

Where

$$
\begin{aligned}
& v_{\mathrm{d} 0}=\gamma_{\mathrm{v}} \frac{\mathrm{v}_{\mathrm{cp}}}{\mathrm{c}} v_{\mathrm{sp}}=\gamma_{\mathrm{v}}\left(1-\frac{\mathrm{v}}{\mathrm{c}}\right) v_{\mathrm{sp}} \\
& v_{\mathrm{d} 0}=\gamma_{\mathrm{v}} \frac{\mathrm{v}_{\mathrm{ca}}}{\mathrm{c}} v_{\mathrm{sa}}=\gamma_{\mathrm{v}}\left(1+\frac{\mathrm{v}}{\mathrm{c}}\right) v_{\mathrm{sa}}
\end{aligned}
$$

Here, $v_{\mathrm{sa}}$ and $v_{\mathrm{sp}}$ are the space frequencies of photons approaching the detector from anti-parallel and parallel directions respectively. While the effective detected frequency $v_{\mathrm{d} 0}$ is the characteristic transition frequency, a photon of frequency $v_{\mathrm{sa}}$ is required from the head-end in a detector moving with velocity $\mathbf{v}$, and a photon of frequency $v_{\mathrm{sp}}$ is required for absorption from the tail-end or parallel direction to induce the characteristic transition. This may be interpreted as an anisotropic change in electron transition energy for a detector moving with velocity v.

The Doppler equations (42) essentially correlate the photon frequency $v_{\mathrm{d} 0}$ required for effecting a particular electron transition in a static detector with the corresponding photon frequencies $v_{\mathrm{sp}}$ and $v_{\mathrm{sa}}$ required for effecting the same electron transition in a moving detector from parallel and anti-parallel directions respectively. As per current interpretation, the anti-parallel photon frequency $v_{\mathrm{sa}}$ is said to be up-shifted to observed or detected frequency $v_{\mathrm{d} 0}$ for the moving detector as per equation (42B). Similarly, the parallel photon frequency $v_{\mathrm{sp}}$ is said to 
be down-shifted to observed or detected frequency $v_{\mathrm{d} 0}$ for the moving detector as per equation (42A). Hence, we might consider $v_{\mathrm{d} 0}$ as the effective absorption or detected frequency for the moving detector. Therefore, the Doppler equations may be viewed as correlations between the pre-absorption space frequencies $\left(v_{\mathrm{sp}}\right.$ or $\left.v_{\mathrm{sa}}\right)$ and their effective absorption or detected frequency $v_{\mathrm{d} 0}$.

\subsection{Combined Doppler Effect due to the motion of Emitter and Detector for Photon beams}

Let us consider an emitter moving along positive $\mathrm{X}$-axis with velocity $\mathbf{u}$ and a detector also moving, ahead of emitter, along positive $\mathrm{X}$-axis with velocity $\mathbf{v}$. Let us assume the emitter emits a beam of photons, which propagates along positive $\mathrm{X}$-axis at velocity $\mathbf{c}$. For $+\mathrm{ve}$ values of $\mathbf{u}, \mathbf{v}$ and $\mathbf{c}$, this case corresponds to parallel emission and parallel detection (equations 35 and $42 \mathrm{~A}$ ), which are interlinked through photon frequency $v_{\mathrm{sp}}$ during its propagation in space between emitter and detector.

$$
\begin{gathered}
v_{\mathrm{sp}}=v_{\mathrm{up}}=\gamma_{\mathrm{u}}\left(1-\frac{\mathrm{u}}{\mathrm{c}}\right) v_{0} \\
v_{\mathrm{d} 0}=\gamma_{\mathrm{v}}\left(1-\frac{\mathrm{v}}{\mathrm{c}}\right) v_{\mathrm{sp}}
\end{gathered}
$$

Here $v_{0}$ is the frequency of photons emitted from a static emitter and $v_{\mathrm{sp}}$ is the space frequency of photons emitted in forward direction from the emitter moving with velocity $\mathbf{u}$. When the photons of space frequency $v_{\mathrm{sp}}$ get absorbed from the tail-end in a detector moving with velocity $\mathbf{v}$, the detected or observed frequency $v_{\mathrm{d} 0}$ is given by equation (44). The corresponding Doppler shift parameter $\mathrm{z}_{\mathrm{pp}}$ for parallel emission and parallel absorption is given by,

$$
1+z_{p p}=\frac{v_{0}}{v_{d 0}}=\frac{v_{0}}{v_{s p}} \frac{v_{\mathrm{sp}}}{v_{d 0}}=\frac{1}{\gamma_{\mathrm{u}}(1-\mathrm{u} / \mathrm{c})} \cdot \frac{1}{\gamma_{\mathrm{v}}(1-\mathrm{v} / \mathrm{c})}=\frac{\sqrt{(1+\mathrm{u} / \mathrm{c})}}{\sqrt{(1-\mathrm{u} / \mathrm{c})}} \cdot \frac{\sqrt{(1+\mathrm{v} / \mathrm{c})}}{\sqrt{(1-\mathrm{v} / \mathrm{c})}}
$$

Similarly, the Doppler shift parameter $z_{a a}$ for anti-parallel emission and anti-parallel absorption will be given by,

$$
1+z_{a a}=\frac{v_{0}}{v_{d 0}}=\frac{v_{0}}{v_{s a}} \frac{v_{\mathrm{sa}}}{v_{d 0}}=\frac{1}{\gamma_{\mathrm{u}}(1+\mathrm{u} / \mathrm{c})} \cdot \frac{1}{\gamma_{\mathrm{v}}(1+\mathrm{v} / \mathrm{c})}=\frac{\sqrt{(1-\mathrm{u} / \mathrm{c})}}{\sqrt{(1+\mathrm{u} / \mathrm{c})}} \cdot \frac{\sqrt{(1-\mathrm{v} / \mathrm{c})}}{\sqrt{(1+\mathrm{v} / \mathrm{c})}}
$$

The Doppler shift parameter $\mathrm{z}$ for any other combination of emitter and detector velocities can be similarly worked out. It is pertinent to note here that the shift in detected frequencies due to the motion of detector, as given by equations (4), (5), (12) and (13) in the case of continuously emitted waves, is identical to the shift in detected frequencies given by equations (42) in case of spontaneously emitted beam of photons. However, the frequency shift caused by the motion of emitter is not the same in the two cases. The frequency shift due to the motion of emitter, as given by equations (2) and (3) for continuously emitted waves, is just the standard Doppler shift adopted in classical theory of the phenomenon. As per this, frequency of continuous waves emitted in a forward direction parallel to the emitter velocity, gets 'up-shifted' or 'blue-shifted' due to the motion of emitter. Similarly, frequency of continuous waves emitted in a backward direction, anti-parallel to the emitter velocity, gets 'down-shifted' or 'red-shifted' due to the motion of emitter. On the other hand, frequency shift due to the motion of emitter for the spontaneously emitted beam of photons, as given by equations (35) and (36), is quite opposite to the one given by the classical theory. As per equation (35), frequency of photons spontaneously emitted in a forward direction parallel to the emitter velocity, gets 'down-shifted' or 'red-shifted' due to the motion of emitter. As per equation (36), frequency of photons spontaneously emitted in a backward direction, anti-parallel to the emitter velocity, gets 'up-shifted' or 'blue-shifted' due to the motion of emitter. The electron transition energy levels also get shifted in the same proportion as the shift in emitted photon frequency.

\section{Tests for Verification of Doppler Effect for Photons}

Most of the tests for verification of Doppler effect conducted so far, have been carried out by using continuously emitted waves. In fact, due to the diverse practical applications of the Doppler effect for continuous waves, tests for its verification are no longer considered necessary. However, specific tests for verification of Doppler effect for spontaneously emitted photons are quite limited. The relativistic Doppler effect is distinguished from the classical Doppler effect by the Lorentz factor $\gamma$ apart from the relative separation or approach velocity. Most of the current relativistic Doppler tests either strive to verify the role of $\gamma$ factor to better accuracy or verify the Doppler effect for spontaneous photon absorption in moving detector or absorber systems. Separate tests for verification of Doppler effect for absolute motion of emitter alone, are generally neither planned nor conducted.

Earliest tests for verification of Doppler effect for spontaneous photon emission from moving emitters were conducted with so called 'canal rays' or positive ion beams (Wien, 1999). However, based on an invalid 
assumption of fast moving ions emitting photons in gas discharge tubes, Stark, (1907) had claimed the verification of Doppler effect by measuring some Doppler shifts in Hydrogen spectrum lines. Although Stark is known to be the pioneer of Stark effect, the effect of electric field on the splitting of emission lines, he overlooked the effect of high electric field on the ionic interaction and emission phenomenon in the observation chamber. Similarly, Batho and Dempster (1932) had overlooked the possibility of fresh ionization of neutral hydrogen molecules during their collision with high speed canal rays and their subsequent motion towards the cathode, before final electron capture. As such, all tests conducted with canal rays to verify Doppler effect in the direction of motion of the emitter could be considered as erroneous or misinterpreted since emitters of light were not moving with uniform speed in one direction.

In the famous experiment by Ives and Stilwell (1938), the displaced Doppler lines are observed corresponding to motion toward and away from the observer, and the effect to be observed is a shift of the center of gravity of the displaced lines with respect to the un-displaced line. Though the scope of Ives and Stilwell test was limited to verify the role of Lorentz $\gamma$ factor in the relativistic Doppler effect, similar other tests are also called Lorentz Invariance (LI) tests or Time dilation tests. In Ives and Stilwell's experiment, the Doppler effect had been studied by measuring the frequency of the emitted photons. However, a major change occurred with the appearance of lasers; the measured quantity became the frequency of the photons absorbed by the atoms in flight in place of their emitted frequencies.

Saathoff, et al. (2011) conducted such LI tests with fast Li ion beams from accelerator storage rings. By treating the high-speed ions as moving detectors or absorbers and optically pumping them with laser beams of measured frequencies in parallel and antiparallel directions, the known electronic transitions were monitored. We may rewrite equations (42A) and (42B) by replacing the detected frequency $v_{\mathrm{d} 0}$ with static absorption frequency $v_{0}$ for transition between selected energy levels and ratio $v / c$ with $\beta$, as optical pumping equations.

$$
\begin{gathered}
v_{0}=\gamma(1-\beta) \nu_{\mathrm{sp}} \\
v_{0}=\gamma(1+\beta) \nu_{\mathrm{sa}} \\
\gamma=\frac{1}{\sqrt{1-\beta^{2}}}
\end{gathered}
$$

Where

Here $v_{\mathrm{sp}}$ is the photon frequency in the laser beam directed along or parallel to the high-speed ion beam and $v_{\mathrm{sa}}$ is the photon frequency in the laser beam directed opposite or antiparallel to the ion beam. One of the laser beams is called pump beam with higher intensity. The fixed frequency say $v_{\mathrm{sa}}$ of the pump beam is in resonance with the ions at the center $\beta_{0}$ of the velocity distribution. The other probe beam is tuned across the resonance. The laserinduced fluorescence is observed with photomultipliers. Due to saturation, a Lamb dip occurs in the Doppler fluorescence background, when the tuning laser beam is resonant at $v_{\mathrm{sp}}$ with the $\beta_{0}$ ions. Under these saturation resonance conditions, multiplying equations (47A) and (47B), we get;

$$
v_{0}^{2}=v_{s p} v_{s a}
$$

This scheme can be generalized to the case where two different transitions with rest frame frequencies $v_{1}$ and $v_{2}$ are used with optical-optical double resonance spectroscopy on the closed $\Lambda$-type three level system. With a fixed frequency, antiparallel laser beam resonant with one of the legs of the $\Lambda$ while scanning the second parallel laser beam over the second leg, fluorescence is observed only when both lasers are resonant with the same ions at the center $\beta_{0}$ of the velocity distribution. In this case, Doppler absorption equations (47A) and (47B) are modified as,

$$
\begin{aligned}
& v_{1}=\gamma(1-\beta) v_{\mathrm{sp}} \\
& v_{2}=\gamma(1+\beta) v_{\mathrm{sa}}
\end{aligned}
$$

Multiplying equations (49A) and (49B), we get;

$$
v_{1} v_{2}=v_{s p} v_{s a}
$$

Botermann, et al. (2014) have confirmed that equations (48) and (50) have been experimentally verified with high accuracy at $\beta=0.338$ thereby validating the Doppler absorption equations (42) and (47). It is a matter of historical misinterpretation that such sophisticated experiments are being regarded as verification of Time dilation or Lorentz Invariance, instead of verification of Doppler absorption equations.

\section{High Astronomical Red Shifts associated with Relativistic Shock Waves}

Cosmological Redshift observed in the radiation from distant celestial objects is believed to be caused by the expansion of space, through spatial stretching of photons. In the geometric interpretation of Gravitation, where matter physically influences the metric and hence the curvature of spacetime, the $4 \mathrm{D}$ spacetime continuum is 
implied to be a physical entity. Fundamentally, matter as a physical entity cannot influence an abstract geometric construct. Sandhu (2011) has shown that the 4D spacetime of GR is not a physical entity but just an abstract mathematical construct with differential scale coordinate system. As such, the expansion of space and the associated Cosmological Redshift are desperate props for attempting plausible interpretation of the observed Astronomical redshifts.

There has been a considerable opposition to the current model of Big Bang Cosmology (Roy Keys, 2002), though without much impact for want of a credible alternative. Halton Arp had presented a large volume of data to show that large quasar redshifts are mostly their intrinsic redshifts and have little correlation with distance (Arp, Burbidge E., \& Burbidge G., 2004). They have collected data to show that some of the high redshift quasars are in fact physically connected with low redshift galaxies (Arp \& Fulton, 2008).

Large-scale structures of galaxies and clusters have been observed over distances spanning at least 150 megaparsecs (Mpc). Galaxy clusters are the largest structures which are known to have reached dynamic equilibrium. Inhomogeneities in the mass distribution can induce motions of galaxies and clusters. Since gravitation is known to operate at such large astronomical distances, in the absence of space expansion, gravitational influence must make the stellar objects move towards each other. When approaching galaxies or clusters collide or merge together, the normal dynamic equilibrium of interacting stellar objects gets disturbed through induced tidal effects (Drake, Kuranz, Miles, Muthsam, \& Plewa, 2009). Dynamic equilibrium can also be disrupted by stellar collisions and internal core disturbances in stellar objects. Such dynamic instabilities, fluctuations or explosions can give rise to strong shock waves (Chevalier, et al. 1980), with accompanying photon emissions.

Shock waves in stellar explosions often become radiation mediated shocks. Such shocks are dominated by radiation energy density rather than thermal energy of matter. An important type of astrophysical shock is the relativistic shock, in which the shock velocity is a significant fraction of the speed of light. Relativistic shocks (Nakar \& Sari, 2012) are theoretically expected in gamma ray bursts, active galactic nucleus jets and in all types of stellar explosions. Since the stellar environment is dominated by plasma, in addition to the rotational motion, the electric and magnetic fields strongly influence the morphology of explosion shocks and, in extreme cases, result in launching jets or highly collimated outflows. Various types of astrophysical shock waves, while propagating towards the observer, will give out strong emissions that are redshifted as per equations (35) and (43).

As discussed in section 3 above, the Doppler shifts in photon frequencies depend on the absolute motions of the photon emitters and detectors. The absolute motion of celestial photon emitters may be split into motion of the center of mass $(\mathrm{CoM})$ of the parent body and local relative motion of the photon emitter with respect to the CoM of the parent body. Accordingly, in view of equation (35), the net Doppler redshift in photons emitted from stellar objects will consist of following main components.

I. Proper Doppler emission redshift due to the absolute velocity of the $\mathrm{CoM}$ of the emitting stellar object towards the observer. This redshift is mainly applicable for emissions from stellar objects in normal dynamic equilibrium.

II. Intrinsic Doppler emission redshift due to the local relative velocity of the photon emitters, with respect to the CoM of parent stellar object, towards the observer. The photons emitted from various types of shock waves propagating towards the observer will show such Intrinsic redshifts.

III. Detector end absorption redshift due to the absolute velocity of the observer/detector parallel to the velocity of photons being absorbed. As per equation (42A), the space frequency of a photon being captured by a moving detector from the tail-end or parallel direction, gets down-shifted or redshifted.

The experimentally detected or observed emission redshift in light received from distant astronomical sources normally consists of all the above mentioned three components but in different proportions. The first component, Proper Doppler emission redshift, is the major or dominant component of the redshift in light received from distant stellar objects that are in stable dynamic equilibrium like our sun. This redshift primarily depicts the absolute approach velocity of the stellar source under observation. The second component, Intrinsic Doppler emission redshift, is the dominant component of the redshift in light received from distant stellar objects that are experiencing disruptive changes in their structure with associated ultra-strong shock waves, as in case of Quasars and Supernovae redshifts. Such intrinsic redshifts (Bell \& Comeau, 2003) are primarily caused by various shock waves moving towards the observer at relativistic speeds while emitting characteristic radiation in different colors. Finally, the Detector end absorption redshift is expected to be a significant component when we want to compute the absolute velocities of astronomical objects from their observed total redshifts. 
However, when light of continuum spectrum coming from a background source passes through a moving cloud of gas or dust before reaching the observer, it may show characteristic absorption lines in the spectrum. These absorption lines will be redshifted if the absorber atoms or molecules are receding from the observer or moving anti-parallel to the incoming light photons. When the absorber atoms or molecules receding from the observer at velocity $\mathrm{v}$ encounter a photon of frequency $v_{\mathrm{sa}}$ approaching head-on or from anti-parallel direction, then as per equation (42B) its frequency will appear upshifted to induce a transition of effective frequency $v_{\mathrm{d} 0}$. That means, from the continuum spectrum of the background light, frequency $v_{\text {sa }}$ will get absorbed to induce the characteristic transition of frequency $v_{\mathrm{d} 0}$, so that the characteristic absorption line of frequency $v_{\mathrm{d} 0}$ will appear redshifted to absorption frequency $v_{\text {sa. }}$.

\section{Summary and Conclusion}

Under certain plausible assumptions, electron orbits can be modeled for simple atomic systems and such studies show that all permissible electron trajectories correspond to elliptical orbits. Simulations of such electron orbits can provide insight into the detailed process of electron energy transitions. From the conservation of energy, momentum and angular momentum, in conjunction with the geometrical model of electron trajectories, we derived the Doppler effect for spontaneously emitted photons that is different from the one used for continuously generated waves. Unfortunately, there is no conclusive experimental evidence available for the verification of the proposed Doppler effect for the spontaneously emitted photons.

The relativistic Doppler effect given by equation (11), fails to account for the separate motions of the emitter or the detector in any Laboratory or universal reference frame. All observations of astronomical redshifts are always interpreted by assuming the incoming radiation to be consisting of continuously emitted waves. Therefore, as per equation (11), the observed astronomical redshift is currently interpreted as a measure of receding velocity of the astronomical source. As such, the widely-observed redshift in most astronomical sources gives the general impression that our universe is continuously expanding outwards. Coupled with the Hubble relation that places high redshift objects at greater distances, the implied highly relativistic receding velocities of some quasar type objects reaffirms the notion of cosmological expansion of space.

However, for the spontaneously emitted photons, as shown in equation (35), the photons emitted in forward direction parallel to the emitter velocity get redshifted. That means, the widely-observed redshift in most astronomical sources must imply that the emission sources are moving towards or approaching the observer and our universe is not expanding outwards. Halton Arp had suspected long ago that most quasars with high redshifts are physically linked with nearby low redshift galaxies and do not follow the Hubble relation for their location distance. The dominant component of their redshift is the Intrinsic redshift associated with relativistic shock waves propagating towards the observer. Hence the proposed Doppler effect for the spontaneously emitted photons drastically changes our perspective of the universe and demolishes the currently accepted model of cosmology.

\section{References}

Arp, H., \& Fulton, C. (2008). A Cluster of High Redshift Quasars with Apparent Diameter 2.3 Degrees. Retrieved from https://arxiv.org/abs/0802.1587v1

Arp, H., Burbidge, E. M., \& Burbidge, G. (2004). The double radio source 3C 343.1: A galaxy-QSO pair with very different redshifts. Astronomy \& Astrophysics, 414(3), L37-L40. Retrieved from http://arxiv.org/abs/astro-ph/0401007v1

Batho, H. F., \& Dempster, A. J. (1932). Doppler Effects in Hydrogen Canal Rays of Uniform Energies. Astrophysical Journal, 75(34), 1932. http://dx.doi.org/10.1086/143352

Bell, M. B., \& Comeau, S. P. (2003). Intrinsic Redshifts and the Hubble Constant. Retrieved from http://arxiv.org/abs/astro-ph/0305060v1

Botermann, B., Bing, D., Geppert, C., Gwinner, G., Hansch, T. W., Huber, G., ... Saathoff, G. (2014). Test of Time Dilation Using Stored Li+ Ions as Clocks at Relativistic Speed. Physical Review Letters, 113(12), 120405. Retrieved from https://arxiv.org/pdf/1409.7951v1.pdf

Chevalier, R. A., Kirshner, R. P., \& Raymond, J. C. (1980). The Optical Emission from a Fast Shock Wave with Application to Supernova Remnants. Astrophysical Journal, Part 1, 235, 186-195. http://dx.doi.org/10.1086/ 157623

Drake, R. P., Kuranz, C. C., Miles, A. R., Muthsam, H. J., \& Plewa, T. (2009). Stellar explosions, instabilities, and turbulence. Physics of Plasmas, 16, 041004 (2009). http://dx.doi.org/10.1063/1.3101816 
Ives, H. E., \& Stilwell, G. R. (1938). An Experimental Study of the Rate of a Moving Atomic Clock. Journal of the Optical Society of America, 28(7), 215-226. https://dx.doi.org/10.1364/JOSA.28.000215

Nakar, E., \& Sari, R. (2012). Relativistic Shock Breakouts- A Variety of Gamma-Ray Flares: From Lowluminosity Gamma-Ray Bursts to Type Ia Supernovae. Astrophysical Journal, 747(2), 15. http://dx.doi.org/10.1088/0004-637X/747/2/88

Roy Keys. (2002). The Top 30 Problems with the Big Bang. Apeiron, 9(2). Retrieved from http://redshift.vif.com/JournalFiles/V09NO2PDF/V09N2tvf.PDF

Roychoudhuri, C., \& Ambroselli, M. (2013, October). Can one distinguish between Doppler shifts due to sourceonly and detector-only velocities? In SPIE Optical Engineering + Applications (pp. 88321E). International Society for Optics and Photonics. http://dx.doi.org/10.1117/12.2018342

Saathoff, G., Reinhardt, S., Bernhardt, B., Holzwarth, R., Udem, Th., Hansch, T. W., ... Gwinner, G. (2011). Testing Time Dilation on Fast Ion Beams. Journal of Physics: Conference Series, 312(10). http://dx.doi.org/10.1088/1742-6596/312/10/102014

Sandhu, G. S. (2009). Fundamental Nature of Matter and Fields. iUniverse Inc. Bloomington, IN, 33 (2009). Retrieved from http://bookstore.iuniverse.com/Products/SKU-000127260/Fundamental-Nature-of-Matterand-Fields.aspx

Sandhu, G. S. (2011). Demystification of the spacetime model of relativity. Physics Essays, 24(1), 85-94 http://dx.doi.org/10.4006/1.3543877

Sandhu, G. S. (2012). Detection of absolute motion through measurement of synchronization offsets. Applied Physics Research, 4(4). http://dx.doi.org/10.5539/apr.v4n4p73

Sandhu, G. S. (2016). Fundamental Invalidity of all Michelson-Morley type Experiments. Applied Physics Research, 8(3). http://dx.doi.org/10.5539/apr.v8n3p45

Stark, J. (1907). On the Radiation of Canal Rays in Hydrogen. Astrophysical Journal, 25, 23. http://dx.doi.org/10. $1086 / 141413$

Wien, K. (1999). 100 Years of Ion Beams: Willy Wien's Canal Rays. Braz. J. Phys., 29(3). http://dx.doi.org/10.1590/S0103-97331999000300002

\section{Copyrights}

Copyright for this article is retained by the author(s), with first publication rights granted to the journal.

This is an open-access article distributed under the terms and conditions of the Creative Commons Attribution license (http://creativecommons.org/licenses/by/4.0/). 